\title{
Limiting reductive stress for treating in-stent stenosis: the heart of the matter?
}

\author{
Judy B. de Haan
}

Baker IDI Heart and Diabetes Institute, Diabetic Complications Division, Oxidative Stress Laboratory, Melbourne, Victoria, Australia.

\begin{abstract}
Restenosis after balloon angioplasty and stenting (BAS) remains an unsolved clinical dilemma for patients with coronary artery disease. A better understanding of the mechanisms that drive this phenomenon is likely to lead to more effective treatments. In this issue of the $J C l$, Ali et al. uncover a critical redox axis with the antioxidant enzyme glutathione peroxidase-1 (CPX1) at its hub and identify potential new therapeutic targets, such as ROS1 tyrosine kinase. This study represents a potential new approach to finding a treatment for BAS, with implications that may extend beyond BAS to other vasculopathies involving vascular remodeling.
\end{abstract}

\section{Vascular injury and drivers of stenosis}

Balloon angioplasty and stenting (BAS) remains the preferred method of treatment for flow-limiting atherosclerosis, far outnumbering coronary artery bypass grafting (1). Unfortunately, stent-mediated intimal hyperplasia, a consequence of vascular smooth muscle cell (VSMC) proliferation, remains a cause of restenosis after BAS and often presents with acute coronary syndrome. Reendothelialization of the damaged vessel has been proposed to reduce intimal hyperplasia; however, current treatment options, such as drugeluting stents, aimed at limiting VSMC proliferation may also inhibit reendothelialization. Reduced reendothelialization leads to an increased risk of thrombosis within the stented vessel, often resulting in devastating clinical consequences (2). Thus, there is a clinical need for improvements in the treatment of flow-limiting atherosclerosis. Recent advances have been made in preclinical models, including the use of mesenchymal stem cells (3) and miRNA-based strategies (4), to assist with reendothelialization. The potential for these new approaches to lead to clinical breakthroughs will likely be expedited once the mechanisms behind BAS-driven hyperplasia are better understood.

Over the past decade, there has been a groundswell of clinical and preclinical evidence that indicates the antioxidant enzyme glutathione peroxidase-1 (GPX1) is involved in the atherogenic process. Clinically, low levels of GPX1 have been associated with an increased risk of cardiovascular events in patients with coronary artery disease, independent of traditional risk factors (5), and GPX1 activity has also been shown to be reduced in excised human plaques (6). Preclinically, plaque analysis in atherosclerosis-prone mice revealed that a lack of GPX1 accelerates plaque formation under both hyperlipidemic (7) and diabetic (8) conditions. While previous studies on GPX1 in vascular injury have focused on its role in atherosclerosis, particularly the involvement of GPX1 in immune cells (9), the role of GPX1 in VSMCs, the drivers of stenosis, has not been characterized. In this issue of the JCI, Ali et al. (10) demonstrate that vascular injury-induced loss of GPX1 alters oxidoreductive stress in VSMCs and promotes in-stent stenosis. This study opens up new frontiers for in-stent stenosis research and therapy and also highlights the need

Related Article: p. 5159

Conflict of interest: The author discloses research support from Reata Pharmaceuticals.

Reference information: J Clin Invest. 2014;124(12):5092-5094. doi:10.1172/JCI79423.

to look further afield at reductive stress when dealing with imbalances in redox events. GPX1 is known to perform important functions in limiting reactive oxygen species-mediated lipid, protein, and DNA damage (11); therefore, the oxidative stress that results from a decline in GPX1 levels has been touted as the mediator of injury. The current report from Ali and colleagues necessitates reevaluation of this paradigm and identifies additional biochemical mechanisms that involve reductive stress and are modulated as a result of GPX1dependent pathways.

\section{GPX1: at the center of stent-} induced pathologic remodeling Using a networks approach to compare coronary vascular gene expression between de novo atherosclerosis and in-stent stenosis patients, Ali et al. (10) identified GPX1 as the network hub gene involved in pathological remodeling following stenting. Evaluation of SNPs and confirmation with GWAS revealed that mutations within GPX1 and the orphan protooncogene receptor tyrosine kinase ROS1 are tightly associated with in-stent stenosis. To elucidate the contribution of GPX1 in the development of stenosis, Ali et al. employed a model of BAS in atherosclerosis-prone (ApoE-deficient) mice lacking GPX1. Compared to animals lacking ApoE alone, mice deficient for both ApoE and GPX1 were more susceptible to BAS-mediated neointimal restenosis. Importantly, the authors determined that ROS1, which drives VSMC proliferation, migration, and survival, is low under physiological conditions, but markedly upregulated after BAS in GPX1-deficient VSMCs. Inhibition of ROS1, either pharmacologically or with siRNA, in GPX1-deficient VSMCs dramatically decreased VSMC expansion, highlighting the possibility that GPX1 mediates stenosis through ROS1 activation.

Importantly, Ali and colleagues discovered that reductive stress, defined as higher levels of reduced glutathione (GSH) 
?

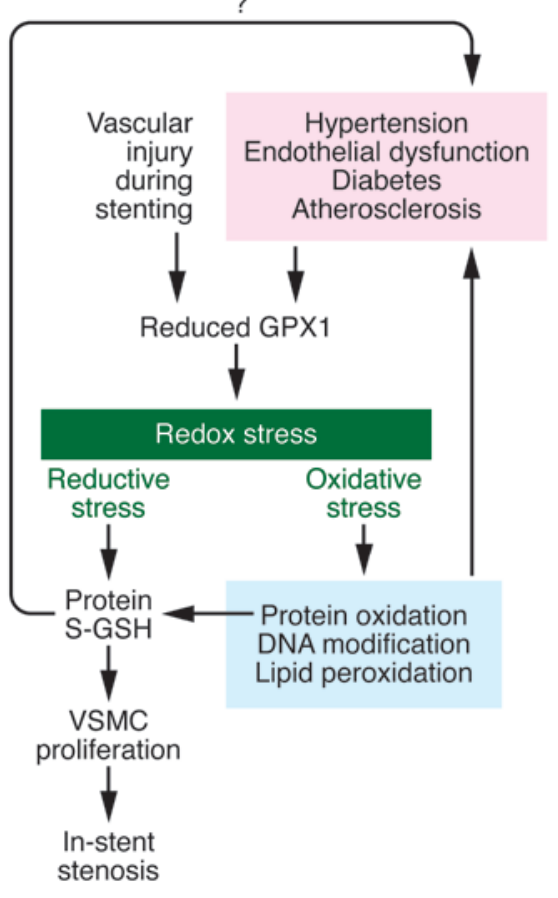

compared with oxidized glutathione (GSSG), is increased after BAS, providing a link between GPX1 and ROS1. The elevated GSH/GSSG ratio most likely occurred as a consequence of upregulation of the GSH-synthesizing enzymes in response to stent-induced vascular injury and the resulting requirement for $\mathrm{GSH}$-dependent DNA synthesis to repair damaged tissue. Moreover, the increase in GSH combined with the reduction in GSH utilization due to the absence of GPX1 leads to the perfect "reductive" storm. Ali and colleagues determined that ROS1 was not a target of $s$-glutathiolation; therefore, they needed to look further afield to determine whether reductive stress affected other proteins. Using peptide mass fingerprinting of ROS1 IP complexes and the knowledge that SHP tyrosine phosphatases regulate ROS1 phosphorylation, the authors identified a physical relationship between ROS1 and SHP-2. Further analysis revealed that specific cysteine sites within SHP-2 are prone to irreversible s-glutathiolation. Computational modeling showed that s-glutathiolation blocked access to the phosphatase active site of SHP-2, thereby rendering it incapable of removing phosphates from ROS1, resulting in sustained phosphorylation and activation of ROS1. Furthermore, Ali and colleagues used their animal model and confirmed the presence and asso-

Figure 1. Loss of GPX1 induces oxidoreductive stress and promotes vascular remodeling. Risk factors for vascular remodeling, such as hypertension, endothelial dysfunction, diabetes, and atherosclerotic plaques, and vascular injury during stenting are associated with reductions in the antioxidant enzyme GPX1. Reductions in GPX1 lead to redox stress, with increases in both reductive stress via GSH and oxidative stress via enhanced production of superoxide $\left(\mathrm{O}_{2}{ }^{-}\right)$, hydrogen peroxide $\left(\mathrm{H}_{2} \mathrm{O}_{2}\right)$, and peroxynitrite (OONO-). Reductive stress in turn increases $\mathrm{s}$-glutathionylation of key proteins, which in VSMCs, leads to their proliferation, migration, and survival, contributing to in-stent stenosis. Oxidative stress modifies DNA, lipids, and proteins and is known to affect pathways associated with key risk factors (pink box) to exacerbate vasculopathies. It is highly likely that reductive stress via s-glutathionylation of important proteins contributes to vascular remodeling associated with these risk factors. In this issue of the $J C l$, Ali et al. (10) identify GPX1 as the network hub gene capable of mediating both reductive and oxidative stress in VSMCs. Perturbations in reductive stress may turn out to be as important, if not more important, than oxidative stress, as demonstrated in in-stent stenosis, with clinical implications that may extend beyond BAS.

ciation of $s$-glutathionylated SHP-2 with ROS1 in post-BAC vessels, suggesting that s-glutathionylated SHP-2 increases ROS1 phosphorylation. Indeed, phosphorylated ROS1 levels were substantially elevated in mice lacking GPX1 following BAS. Importantly, ROS1 inhibition with the ROS1 inhibitor crizotinib or deglutathiolation of SHP-2 abolished GPX1-mediated increases in VSMC proliferation, but kept endothelialization intact.

\section{Conclusions and future directions}

Together, this study by Ali et al. has resulted in several important findings that expand our understanding to include previously unidentified molecular pathways now implicated in stenosis. These findings are likely to have clinical implications. First, the finding that reductive stress over and above oxidative stress is the driver of BAS may explain the failure of antioxidants in clinical trials of vascular remodeling $(12,13)$. Indeed, traditional antioxidants such as vitamins $\mathrm{E}$ and $\mathrm{C}$ would have no effect on stenosis for several reasons, most notably an inability to lessen reductive stress. Future "antioxidant" studies should be directed toward the GSH-synthesizing and -utilizing enzymes as a way to lessen reductive stress associated with BAS. Blocking s-glutathionylation by lowering GSH levels seems counterintuitive, but, as shown by Ali and colleagues, too much of a supposedly good thing (GSH) is not necessarily beneficial. Second, the identification of tyrosine kinase ROS1 as a novel mediator of vascular remodeling provides an attractive candidate for drug intervention. Ali et al. used the ROS1 inhibitor crizotinib; however, this inhibi- tor is known to affect anaplastic lymphoma kinase (ALK) and c-MET/hepatocyte growth factor receptor (HGFR) tyrosine kinase. Future efforts need to focus on the development of more specific ROS1 inhibitors. Whether these inhibitors will have implications for vascular remodeling beyond BAS is currently unknown. Current clinical trials with crizotinib are focused around anticancer treatments for non-small cell lung carcinoma (NSCLC), lymphoma, neuroblastoma, and other advanced solid tumors; therefore, the results of this study are expected to extend the use of this drug class beyond cancer therapy into the field of vascular remodeling. It is possible that the use of such drugs for atherosclerosis-associated vascular remodeling may be limited, given that crizotinib did not reduce atherosclerosis in mice. On the other hand, it should be noted that this action was only investigated at the aortic sinus, a region that is known to be less amenable to drug interventions (14-16). Clearly, further studies are required to test the efficacy of ROS1 inhibition as an antiatherogenic treatment. A further caveat is that this type of inhibitor appears specific for VSMCs, and reduced VSMC proliferation in atherosclerosis has been implicated in fibrous cap weakening, which leads to a more rupture-prone atherosclerotic plaque. Therefore, use of this drug class in this context may not be advantageous. Lack of GPX1 has, however, been shown to contribute to vascular remodeling of the intima and subsequent vascular stiffening in aging vessels with accompanying reductive modifications of eNOS (17). Indeed, targeting reductive stress in aging may be a novel approach to alleviating hypertension-associated endo- 
thelial dysfunction. Third, reendothelialization of stented vessels is a desired outcome to reduce thrombotic events. Current treatment options for drug-eluting stents include the mTOR inhibitor rapamycin. Ali et al. demonstrated that rapamycin markedly reduced reendothelialization in the BAS model of in-stent stenosis, while, importantly, crizotinib did not. The finding that ROS1 inhibitors do not limit reendothelialization makes this class of drug even more attractive than currently available molecules used in drug-eluting stents. Fourth, the identification of two SNPs with point mutations in GPX1 and ROS1 as closely associated and highly ranked for the prediction of in-stent stenosis bodes well for future personalized medicine where risk assessments can be made prior to angioplasty. This type of assessment would allow for the use of drug-eluting stents that specifically target this redox axis to limit instent stenosis in patients with mutations in GPX1 and/or ROS1.

Finally, GPX1 has been shown to decrease in conditions such as diabetes (18) and atherosclerosis (6). Reductive stress, via the pathways identified by Ali and colleagues, may be important possibly even more important than oxidative stress - in mediating the effects associated with these diseases. Thus, it is time to reassess the redox paradigm and to appreciate that one should not focus just on oxidative stress, but also consider the pathways that are altered as the result of reductive stress and induced by increased GSH as a result of GPX1 deficiency (Figure 1).

\section{Acknowledgments}

J.B. de Haan would like to acknowledge the critical reading and constructive input of Mark E. Cooper, chief scientific officer, Baker IDI Heart and Diabetes Institute, Melbourne, Victoria, Australia. J.B. de Haan acknowledges funding support from Reata Pharmaceuticals Inc.

Address correspondence to: Judy B. de Haan, Baker IDI Heart and Diabetes Institute, Diabetic Complications Division, Oxidative Stress Laboratory, PO Box 6492, Melbourne, Victoria, 3004, Australia. E-mail:judy.dehaan@bakeridi.edu.au.

1. Henderson RA, Timmis AD. Almanac 2011: stable coronary artery disease. An editorial overview of selected research that has driven recent advances in clinical cardiology. Heart. 2011;97(19):1552-1559.

2. Yildiz M, Yildiz B, Gursoy MO, Akin I. Recent developments in drug-eluting coronary stents. Cardiovasc Hematol Disord Drug Targets. 2014;14(3):220-224.

3. Kim AK, et al. Inhibitory effects of mesenchymal stem cells in intimal hyperplasia after balloon angioplasty. J Vasc Surg. 2014:S07415214(14)01624-3.

4. Santulli G, et al. A selective microRNAbased strategy inhibits restenosis while preserving endothelial function. J Clin Invest. 2014;124(9):4102-4114.

5. Blankenberg S, et al. Glutathione peroxidase 1 activity and cardiovascular events in patients with coronary artery disease. $\mathrm{N} \mathrm{Engl} \mathrm{JMed}$. 2003;349(17):1605-1613.

6. Lapenna D, et al. Glutathione-related antioxidant defenses in human atherosclerotic plaques. Circulation. 1998;97(19):1930-1934.

7. Torzewski M, et al. Deficiency of glutathione peroxidase-1 accelerates the progression of atherosclerosis in apolipoprotein E-deficient mice. Arte- rioscler Thromb Vasc Biol. 2007;27(4):850-857.

8. Lewis P, et al. Lack of the antioxidant enzyme glutathione peroxidase- 1 accelerates atherosclerosis in diabetic apolipoprotein E-deficient mice. Circulation. 2007;115(16):2178-2187.

9. Cheng $\mathrm{F}$, et al. Impact of glutathione peroxidase-1 deficiency on macrophage foam cell formation and proliferation: implications for atherogenesis. PLoS One. 2013;8(8):e72063.

10. Ali ZA, et al. Oxido-reductive regulation of vascular remodeling by receptor tyrosine kinase ROS1. J Clin Invest. 2014;124(12):5159-5174.

11. Brigelius-Flohe R. Tissue-specific functions of individual glutathione peroxidases. Free Radic Biol Med. 1999;27(9-10):951-965.

12. Yusuf S, Dagenais G, Pogue J, Bosch J, Sleight P. Vitamin E supplementation and cardiovascular events in high-risk patients. The Heart Outcomes Prevention Evaluation Study Investigators. N Engl J Med. 2000;342(3):154-160.

13. Sesso HD, et al. Vitamins $\mathrm{E}$ and $\mathrm{C}$ in the prevention of cardiovascular disease in men: the Physicians' Health Study II randomized controlled trial. JAMA. 2008;300(18):2123-2133.

14. VanderLaan PA, Reardon CA, Getz GS. Site specificity of atherosclerosis: site-selective responses to atherosclerotic modulators. Arterioscler Thromb Vasc Biol. 2004;24(1):12-22.

15. Chew P, et al. Site-specific antiatherogenic effect of the antioxidant ebselen in the diabetic apolipoprotein E-deficient mouse. Arterioscler Thromb Vasc Biol. 2009;29(6):823-830.

16. Witting PK, Pettersson K, Letters J, Stocker R. Site-specific antiatherogenic effect of probucol in apolipoprotein E-deficient mice. Arterioscler Thromb Vasc Biol. 2000;20(8):E26-33.

17. Oelze M, et al. Glutathione peroxidase-1 deficiency potentiates dysregulatory modifications of endothelial nitric oxide synthase and vascular dysfunction in aging. Hypertension. 2014;63(2):390-396.

18. Hamanishi T, et al. Functional variants in the glutathione peroxidase-1 (GPx-1) gene are associated with increased intima-media thickness of carotid arteries and risk of macrovascular diseases in japanese type 2 diabetic patients. Diabetes. 2004;53(9):2455-2460. 